\title{
Magnetic Properties of Mn-Doped Amorphous $\mathrm{SiO}_{2}$ Matrix
}

\author{
D. Milivojevićc ${ }^{a}$, B. Babić-Stojićc $c^{a, *}$, V. Jokanović ${ }^{a}$, Z. Jagličićc ${ }^{b}$ And D. MakoveC ${ }^{c}$ \\ ${ }^{a}$ Vinča Institute of Nuclear Sciences, University of Belgrade, P.O.Box 522, 11001 Belgrade, Serbia \\ ${ }^{b}$ Institute of Mathematics, Physics and Mechanics, Jadranska 19, 1000 Ljubljana, Slovenia \\ ${ }^{c}$ Jožef Stefan Institute, Department for Materials Synthesis, Jamova 39, 1000 Ljubljana, Slovenia
}

Samples of Mn-doped amorphous $\mathrm{SiO}_{2}$ matrix with manganese concentration 0.7 and 3 at.\% have been prepared by a sol-gel method. Transmission electron microscopy analysis has shown that the samples contain agglomerates of amorphous silica particles $10-20 \mathrm{~nm}$ in size. Two types of Mn-rich particles are dispersed in silica matrix, smaller nanoparticles with dimensions between 3 and $10 \mathrm{~nm}$, and larger crystalline areas consisting of aggregates of the smaller nanoparticles. High-temperature magnetic susceptibility reveals that dominant magnetic phase at higher temperatures is $\lambda-\mathrm{MnO}_{2}$. At temperatures below $T_{\mathrm{C}}=43 \mathrm{~K}$ strong ferrimagnetism originating from the minor $\mathrm{Mn}_{3} \mathrm{O}_{4}$ phase masks the relatively weak magnetism of $\lambda-\mathrm{MnO}_{2}$. Magnetic field dependence of the maximum in the zero-field-cooled magnetization for both the samples in the vicinity of $40 \mathrm{~K}$, and a frequency shift of the real component of the AC magnetic susceptibility in the sample with 3 at.\% Mn suggest that the magnetic moments of the smaller $\mathrm{Mn}_{3} \mathrm{O}_{4}$ nanoparticles with dimensions below $10 \mathrm{~nm}$ are subject to thermally activated blocking process just below the Curie temperature $T_{\mathrm{C}}$. The low-temperature maximum in the zero-field-cooled magnetization observed for both the samples below $10 \mathrm{~K}$ indicates possible spin glass freezing of the magnetic moments in the geometrically frustrated $\mathrm{Mn}$ sublattice of the $\lambda-\mathrm{MnO}_{2}$ crystal structure.

PACS: 75.50.Lk, 75.47.Lx, 75.50.Tt

\section{Introduction}

Manganese oxide materials have received much attention because of their importance in technological applications such as catalysts, sensors, new magnetic materials [1]. Among them, manganese dioxide has attracted considerable interest because of possibility of numerous applications in catalysis, ion exchange, magnetoresistance devices and particularly energy storage devices $[2-$ 4]. Several polymorphs of $\mathrm{MnO}_{2}$ has been identified so far, $\alpha, \beta, \gamma$ and $\lambda$ types. Low dimensional Mn-oxide structures greatly affect their physical and chemical properties and improve potential of these materials for applications in various fields. In this work two manganese oxides in the Mn-rich particles embedded in the amorphous $\mathrm{SiO}_{2}$ matrix were identified, major phase $\lambda-\mathrm{MnO}_{2}$ and minor phase $\mathrm{Mn}_{3} \mathrm{O}_{4} \cdot \lambda-\mathrm{MnO}_{2}$ is a metastable form of manganese dioxide whose thermodynamically stable structure is $\beta-\mathrm{MnO}_{2}$ [5]. $\lambda-\mathrm{MnO}_{2}$ phase is usually obtained by removal of $\mathrm{Li}$ from $\mathrm{LiMn}_{2} \mathrm{O}_{4}$ by acid leaching or by electrochemical procedure $[5,6]$, At higher temperatures $\mathrm{Li}_{x} \mathrm{Mn}_{2} \mathrm{O}_{4}$ spinel has paramagnetic properties. At lower temperatures a complex antiferromagnetic (AFM) ordering in $\lambda-\mathrm{MnO}_{2}$ [5], and very complex magnetic ordering in $\mathrm{LiMn}_{2} \mathrm{O}_{4}$ [7] were reported. In addition, spin glass behavior was also observed at low-temperatures in $\lambda-\mathrm{MnO}_{2}, \mathrm{LiMn}_{2} \mathrm{O}_{4}$ and $\mathrm{Li}_{2} \mathrm{Mn}_{2} \mathrm{O}_{4}[6] . \mathrm{Li}_{x} \mathrm{Mn}_{2} \mathrm{O}_{4}$ mate-

\footnotetext{
* corresponding author; e-mail: babic@vinca.rs
}

rial has attracted a wide attention because of its utilization as cathode material for rechargeable Li batteries [34]. In the samples studied in the present work $\mathrm{Mn}_{3} \mathrm{O}_{4}$ structure as a minor phase was found to exist simultaneously with the $\lambda-\mathrm{MnO}_{2}$ phase. The synthesis procedure enabled us to obtain $\mathrm{Mn}_{3} \mathrm{O}_{4}$ nanocrystallites with dimensions below $10 \mathrm{~nm}$ and to study their magnetic properties characteristic to very small nanoparticles.

\section{Experimental details}

The silica sol with very strictly determined size and uniformity of silica sol particles was synthesized using a water glass solution of $\mathrm{Na}_{2} \mathrm{O} \cdot 3 \mathrm{SiO}_{2}$ with the silica module $\mathrm{SiO}_{2} / \mathrm{Na}_{2} \mathrm{O}$ ratio of 3.75 . Then, the second solution with previously stoichiometrically adjusted quantities of $\mathrm{Mn}\left(\mathrm{NO}_{3}\right)_{2} \cdot 6 \mathrm{H}_{2} \mathrm{O}$ salt was added to silica sol solution equivalent to content of 1 and 5 at.\% Mn (nominal manganese concentrations).

The content of manganese in the two samples determined by atomic absorption method is $6.36 \mathrm{mg}$ and 27.34 mg per gram of the sample which corresponds to 0.7 and 3 at.\% of Mn respectively. Powder X-ray diffraction (XRD) spectra were recorded on a Philips PW 1050 diffractometer using $\mathrm{Cu} \mathrm{K} \alpha$ radiation. For transmission electron microscopy (TEM) measurements the samples were deposited on a copper-grid-supported perforated transparent carbon foil. High-resolution transmission electron microscopy (HRTEM) and (semi)quantitative energy dispersive X-ray spectroscopy (EDXS) were car- 
ried out using a field-emission electron-source transmission electron microscope JEOL $2010 \mathrm{~F}$ operated at $200 \mathrm{kV}$. The microscope was equipped with an energy dispersive X-ray spectrometer (LINK ISIS EDS 300). The magnetization measurements were performed on a SQUID magnetometer (MPMS XL-5, Quantum Design) equipped with an $\mathrm{AC}$ option. The $\mathrm{DC}$ magnetization, measured at applied magnetic fields from 50 to 10000 Oe under zero-field-cooled (ZFC) and field-cooled (FC) regime, was recorded in the temperature range from 2 to $300 \mathrm{~K}$. The AC magnetization measurements were made under an AC exciting field with amplitude of 6.5 Oe using different frequencies in the range 1-1488 Hz.

\section{Results and discussion}

XRD spectrum of the sample with 3 at.\% $\mathrm{Mn}$ is presented in Fig. 1. The XRD pattern is characterized by a broad band with a maximum at $2 \theta=21.9^{\circ}$. The shape of the diffractogram and position of the wide band correspond to amorphous silica [8]. No separated narrow XRD lines were observed.

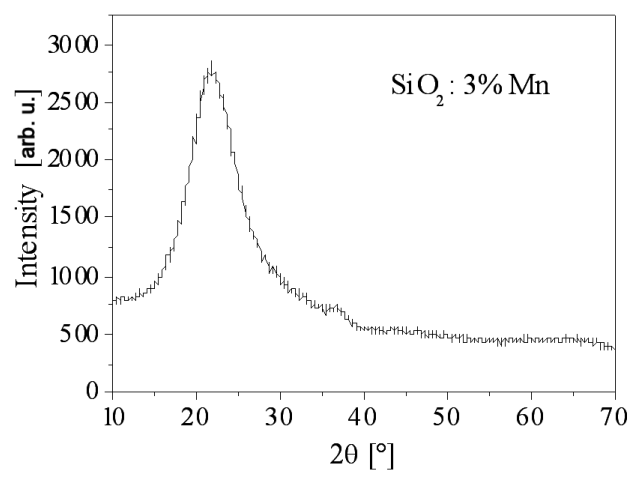

Fig. 1. X-ray diffraction pattern of the $\mathrm{SiO}_{2}: 3$ at.\% Mn sample.

TEM image of the sample with 3 at.\% $\mathrm{Mn}$ is shown in Fig. 2a. This image reveals that the sample contains agglomerates of globular amorphous silica particles of approximately $10-20 \mathrm{~nm}$ in size. EDXS spectra taken from the majority of the individual silica particles showed only $\mathrm{Si}$ and $\mathrm{O}$ suggesting that $\mathrm{Mn}$ is segregated inside the silica matrix. Inspection of the sample using HRTEM showed two types of Mn-rich nanoparticles dispersed inside the silica matrix:

(i) Smaller Mn-rich nanoparticles with sizes between 3 and $10 \mathrm{~nm}$ (marked with circles in Fig. 2b). The nanoparticles are crystalline, as can be seen from regular periodicity of lattice fringes in the HRTEM images (Fig. 2c).

(ii) Rarely, larger crystalline areas, most probably consisting of aggregates of the smaller Mn-rich nanoparticles, were also observed, Fig. 2d.

Some interplanar lattice spacings have been measured for the dispersed nanoparticles and those inside the larger crystalline areas: $2.1 \AA, 2.3 \AA, 2.47 \AA, 2.7 \AA$, and $4.1 \AA$.

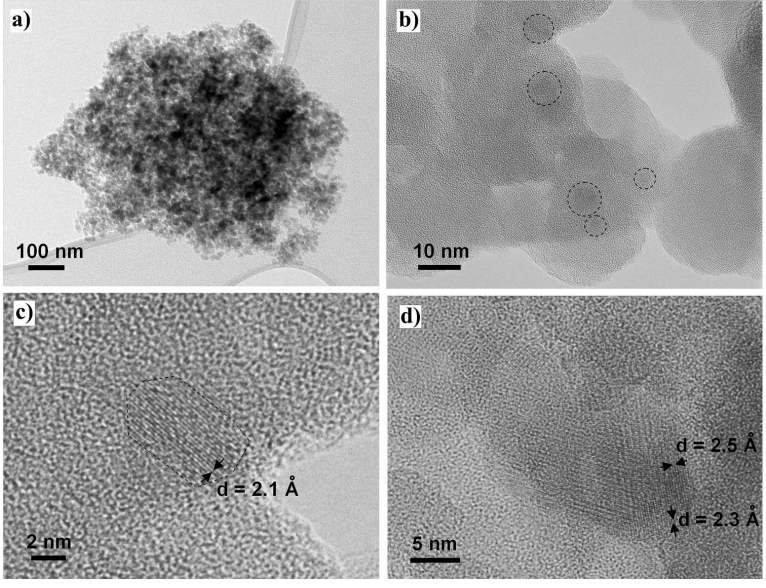

Fig. 2. TEM and HRTEM images of the sample with 3 at.\% Mn: a) agglomerate of the amorphous silica particles, b) individual nanocrystalline particles dispersed in the amorphous silica matrix are marked with circles, c) enlarged area showing the nanocrystalline particle marked with a circle in the upper part of Fig. 2b), d) HRTEM images of the larger crystalline area composed of several smaller Mn-rich nanoparticles inside the amorphous silica matrix.
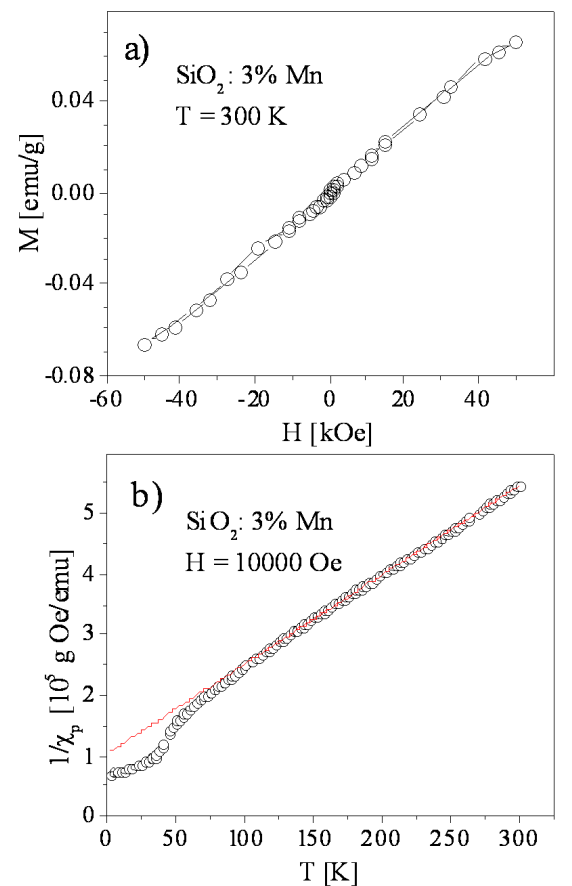

Fig. 3. a) Magnetic field dependent magnetization for the sample with 3 at. $\% \mathrm{Mn}$ at $T=300 \mathrm{~K}$, b) inverse high-temperature paramagnetic susceptibility for the sample with 3 at. \% Mn at the magnetic field of 10000 Oe. The solid curve represents fit of the dependence (1) to the experimental data. 
High-temperature magnetization measured as a function of magnetic field at different temperatures in the range $100-300 \mathrm{~K}$ for the sample with 3 at.\% Mn shows that this dependence is not strictly linear. The $M$ versus $H$ dependence at high $T$ exhibits hysteresis behavior, as can be seen in Fig. 3a which presents the $M(H)$ curve at $T=300 \mathrm{~K}$. We have attributed this hysteresis behavior to a ferromagnetic (FM) component, so that the total magnetization can bee described as $M(T, H)=$ $\chi_{p}(T) H+M_{F M}$, where $\chi_{p}(T)$ is the paramagnetic susceptibility, $H$ is the magnetic field and $M_{F M}$ is the hightemperature ferromagnetic component of magnetization. The ferromagnetic component $M_{F M}$ was estimated as intercept of the linear part of the total magnetization $M$. Paramagnetic susceptibility was then obtained by subtraction of ferromagnetic component from the total magnetization. The paramagnetic susceptibility of the sample with 3 at.\% Mn so evaluated and corrected for diamagnetic susceptibility of silica is shown in Fig. 3b for the field of 10000 Oe. We find that $\chi_{p}(T)$ follows the Curie-Weiss dependence,

$$
\chi_{p}(T)=\frac{C}{T-\theta},
$$

where $C$ is the Curie-Weiss constant and $\theta$ is the CurieWeiss temperature. The best fit parameters for the sample with 3 at.\% Mn in the temperature range 200-300 $\mathrm{K}$ are obtained as $C=6.8 \times 10^{-4} \mathrm{emu} \mathrm{K} / \mathrm{g}_{\text {sample }} \mathrm{Oe}$ which corresponds to $C=1.36 \mathrm{emu} \mathrm{K} / \mathrm{mol}(\mathrm{Mn}) \mathrm{Oe}$ and $\theta=-(70 \pm 5) \mathrm{K}$ indicating AFM interactions between the Mn ions. The FM component of magnetization observed in the high-temperature region is of the order of $1 \times 10^{-3} \mathrm{emu} / \mathrm{g}_{\text {sample }}$ and is estimated to be about $5 \%$ of the total magnetization at the field of 10000 Oe. Until now room temperature FM has been observed in nonmagnetic oxide nanoparticles such as $\mathrm{CeO}_{2}, \mathrm{Al}_{2} \mathrm{O}_{3}$, $\mathrm{ZnO}$ [9]. It was assumed that the origin of the room temperature FM in the oxide nanoparticles might be the exchange interaction between localized electron spins resulting from oxygen vacancies at the surface of the nanoparticles. Thin films of $\mathrm{HfO}_{2}$ have been also reported to exhibit the room temperature FM in the absence of any doping. However, there is a conflicting report attributing the ferromagnetism in $\mathrm{HfO}_{2}$ to possible iron contamination [10]. Origin of the high-temperature FM component of the magnetization in our sample $\mathrm{SiO}_{2}$ : 3 at.\% $\mathrm{Mn}$ is unclear. We cannot exclude the possibility that some sort of FM impurity was present in the starting material used for the silica sol synthesis. The effective magnetic moment per $\mathrm{Mn}$ ion estimated from the Curie constant $C=N \mu_{\text {eff }}^{2} / 3 k_{\mathrm{B}}$ is found to be $\mu_{\text {eff }}=3.3 \mu_{\mathrm{B}} / \mathrm{Mn}$. The values of $C$ and $\mu_{\text {eff }}$ obtained for the sample with 3 at.\% $\mathrm{Mn}$ are somewhat smaller than the theoretical spin-only values for $\mathrm{Mn}^{4+}$ with spin $S=3 / 2, C\left(\mathrm{Mn}^{4+}\right)=1.87 \mathrm{emu} \mathrm{K} / \mathrm{mol}(\mathrm{Mn}) \mathrm{Oe}$ and $\mu_{\text {eff }}\left(\mathrm{Mn}^{4+}\right)=3.87 \mu_{\mathrm{B}}$. Calculated spin-only values of $C$ and $\mu_{\text {eff }}$ for the $\mathrm{Mn}^{3+}$ and $\mathrm{Mn}^{2+}$ ions are considerably higher than those obtained in our experiment. The experimental values of $C$ and $\mu_{\text {eff }}$ obtained for the 3 at.\% Mn sample suggest that dominant magnetic phase at high-temperatures has a form of manganese dioxide. Tetragonal $\beta$ phase of $\mathrm{MnO}_{2}$ exhibits a phase transition at $T_{N} \approx 92 \mathrm{~K}$ with $\mathrm{AFM}$ ordering below $T_{N}$, and CurieWeiss temperature dependence of the magnetic susceptibility above $200 \mathrm{~K}$ with the Curie-Weiss temperature of $-792 \mathrm{~K}$ [11]. Tetragonal $\alpha$ phase and orthorhombic $\gamma$ phase of $\mathrm{MnO}_{2}$ obey the classical Curie-Weiss law above $150 \mathrm{~K}$ with the Curie-Weiss temperature of about $-300 \mathrm{~K}$ and $-180 \mathrm{~K}$ respectively $[12,13]$

Magnetic characteristics of the sample with 3 at.\% Mn observed at high-temperatures resemble the properties of $\lambda-\mathrm{MnO}_{2}$. There is no magnetic phase transition above $50 \mathrm{~K}$, as can be seen in Fig. 4, and the Curie-Weiss temperature $\theta=-70 \mathrm{~K}$ is much lower than that detected in the $\alpha, \beta$ and $\gamma$ phase of $\mathrm{MnO}_{2}$. The XRD spectrum of the 3 at.\% Mn sample does not exhibit the XRD lines corresponding to $\lambda-\mathrm{MnO}_{2}$ (Fig. 1). The HRTEM images, however, enabled us to measure several interplanar lattice spacings. Taking into consideration the limited accuracy of measurements, the spacings can be attributed to the cubic, spinel-type crystal structure of $\lambda-\mathrm{MnO}_{2}: 2.47 \AA$ matching the $d$-value of (311) planes, $2.3 \AA$ matching the $d$-value of (222) planes, $2.1 \AA$ matching the $d$-value of (400) planes and $4.1 \AA$ matching the $d$-value of (200) planes.

$\lambda-\mathrm{MnO}_{2}$ form of manganese dioxide has been studied so far by several authors. The $\lambda-\mathrm{MnO}_{2}$ bulk sample with composition $\mathrm{Li}_{0.10} \mathrm{MnO}_{2}$ prepared by acid treatment of $\mathrm{LiMn}_{2} \mathrm{O}_{4}$ showed the Curie-Weiss behavior in the high-temperature susceptibility with the Curie constant $1.97 \mathrm{emu} \mathrm{K} / \mathrm{mol}(\mathrm{Mn}) \mathrm{Oe}\left(\mu_{\mathrm{eff}}=3.97 \mu_{\mathrm{B}}\right)$ and CurieWeiss temperature of $104 \mathrm{~K}$ [5]. In the $\lambda-\mathrm{MnO}_{2}$ sample with composition $\mathrm{Li}_{0.07} \mathrm{Mn}_{2} \mathrm{O}_{4}$ prepared by electrochemical cycling of $\mathrm{LiMn}_{2} \mathrm{O}_{4}$ the high-temperature susceptibility exhibited the Curie-Weiss dependence with the Curie-Weiss temperature of $-74 \mathrm{~K}$ and Curie constant $3.23 \mathrm{emu} \mathrm{K} / \mathrm{mol}(\mathrm{Mn})$ Oe $\left(\mu_{\mathrm{eff}}=3.60 \mu_{\mathrm{B}}\right)[6]$, which is also below the theoretical spin-only value of $3.87 \mu_{\mathrm{B}}$ for $\mathrm{Mn}^{4+}$. For our sample with 3 at.\% Mn, where the presence of $\mathrm{Li}$ was not detected, the possible reason of the lower value of the parameter $C$ and consequently of $\mu_{\text {eff }}$ compared to the spin-only value for $\mathrm{Mn}^{4+}$ could be somewhat higher value of manganese concentration determined by atomic absorption method (3 at.\% Mn) compared to that actually present in this sample.

In contrast to the results of Greedan et al. [5]. who observed long range AFM order by neutron diffraction below $T_{N}=32 \mathrm{~K}$ in their $\lambda-\mathrm{MnO}_{2}$ sample which was also detected as a maximum in the magnetic susceptibility around $30 \mathrm{~K}$, in our samples with 0.7 and 3 at.\% $\mathrm{Mn}$ there is no peak in the ZFC and FC magnetization in the vicinity of $30 \mathrm{~K}$ (Fig. 4). Better evidence for the absence of the critical temperature near $30 \mathrm{~K}$ can be seen in the first derivative of the product $M T$ with respect to temperature, $\partial(M T) / \partial T$, where $M$ is the ZFC magnetization, which is presented in Figs. 5 and 6 for the samples with 0.7 and 3 at.\% Mn respectively. There is no peak 
in the $\partial(M T) / \partial T$ versus $T$ plot in the vicinity of $30 \mathrm{~K}$ for both the samples. However, we observe another specific property in the samples at low-temperatures which can be seen in a form of weak peaks at about $9 \mathrm{~K}$ in the ZFC magnetization of the samples under the field of 1000 Oe (Fig. 4), as a peak at $8.4 \mathrm{~K}$ in the $\partial(M T) / \partial T$ versus $T$ plot for the 0.7 at.\% Mn sample (Fig. 5), and as a very weak peak at $8.7 \mathrm{~K}$ in the $\partial(M T) / \partial T$ versus $T$ dependence for the 3 at.\% Mn sample (Fig. 6). The low- temperature maximum appears to be magnetic field dependent which can be seen from the position of the peaks in the ZFC magnetization for the sample with 3 at.\% Mn detected at 9 and $5.7 \mathrm{~K}$ under the fields of 1000 and 10000 Oe respectively (Fig. 4, inset).

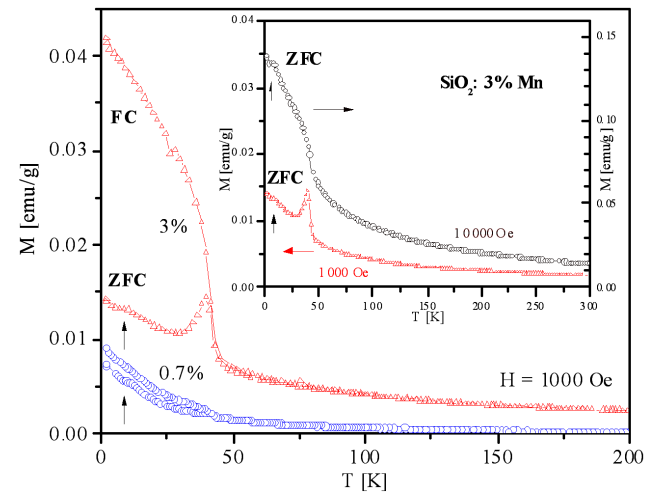

Fig. 4. Temperature dependence of the magnetization under the ZFC and $\mathrm{FC}$ conditions for the 0.7 and 3 at.\% Mn samples at the magnetic field of 1000 Oe. The arrows indicate the positions of the low-temperature peaks in the ZFC magnetization at about $9 \mathrm{~K}$ for both the samples. Inset: ZFC magnetization as a function of temperature for the sample with 3 at. $\% \mathrm{Mn}$ measured at the magnetic fields of 1000 and 10000 Oe: The arrows in the inset indicate the positions of the low-temperature peaks recorded at 9 and $5.7 \mathrm{~K}$ under the fields of 1000 and 10000 Oe respectively.

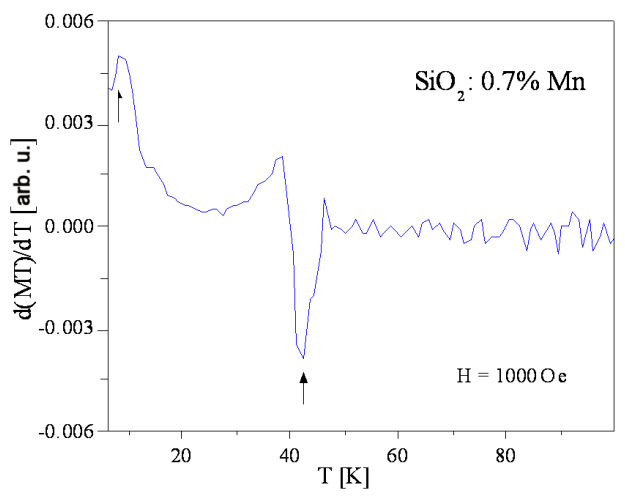

Fig. 5. $\partial(M T) / \partial \mathrm{T}$ for the sample with 0.7 at.\% Mn at the magnetic field of 1000 Oe. The position of the minimum at $42.4 \mathrm{~K}$ and of the low-temperature peak at $8.4 \mathrm{~K}$ are denoted by arrows.

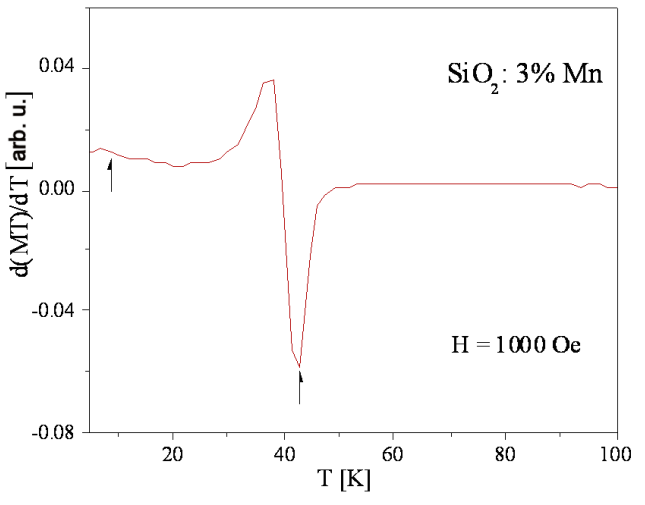

Fig. 6. $\partial(M T) / \partial T$ for the sample with 3 at. $\% \mathrm{Mn}$ at the magnetic field of 1000 Oe. The position of the minimum at $43 \mathrm{~K}$ and of the low-temperature peak at $8.7 \mathrm{~K}$ are denoted by arrows.

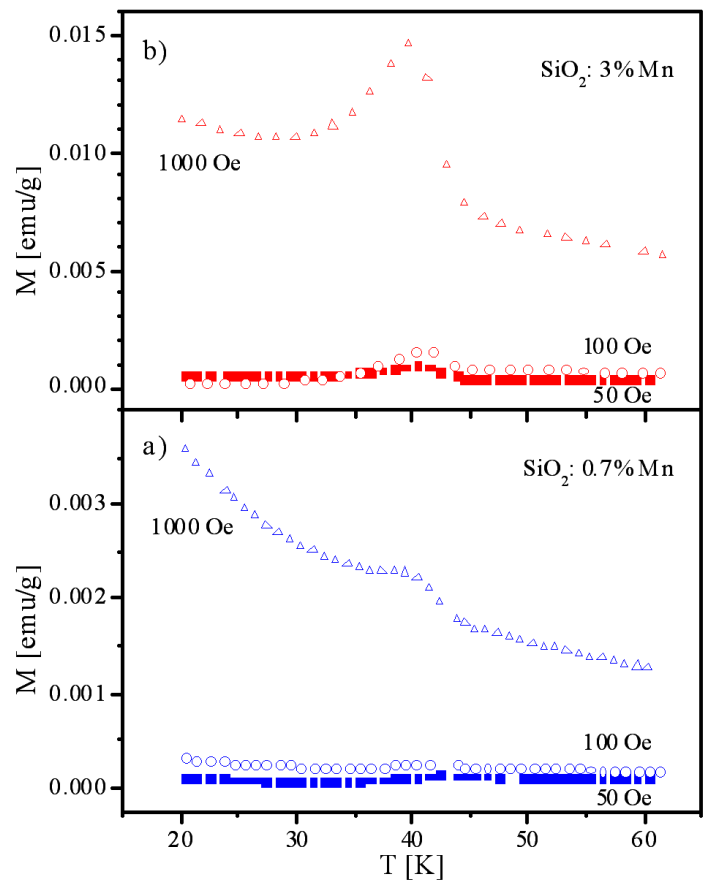

Fig. 7. ZFC magnetization versus temperature at the magnetic fields of 50, 100 and 1000 Oe for the samples with: a) 0.7 at.\% Mn, b) 3 at.\% Mn.

The real part $\chi^{\prime}$ of the AC magnetic susceptibility for the 3 at.\% Mn sample measured as a function of temperature at different frequencies exhibits a broad maximum of low intensity centered at about $14 \mathrm{~K}$ (Fig. 8). Appearance of the peaks in the ZFC magnetization and in the $\partial(M T) / \partial T$ versus $T$ dependence between 5 and $10 \mathrm{~K}$ for both the samples, as well as a broad maximum in the real part of the AC susceptibility observed for the 3 at. $\% \mathrm{Mn}$ sample at about $14 \mathrm{~K}$ could be attributed to a spin glass freezing of the Mn magnetic moments in the $\lambda-\mathrm{MnO}_{2}$ phase of the samples. These properties are 


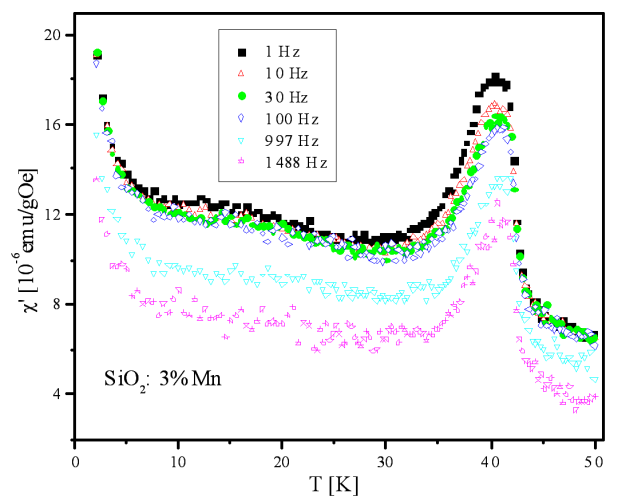

Fig. 8. Temperature dependence of the real part $\chi^{\prime}$ of the AC magnetic susceptibility for the sample with 3 at.\% $\mathrm{Mn}$ at different frequencies in the range 1-1488 $\mathrm{Hz}$.

consistent with geometrical frustration of the manganese sublattice in the $\lambda-\mathrm{MnO}_{2}$ crystal structure [5]. Spin glass behavior was also observed in the $\lambda-\mathrm{Li}_{0.07} \mathrm{Mn}_{2} \mathrm{O}_{4}$ sample at temperatures below $16 \mathrm{~K}[6]$.

The ZFC and FC magnetizations of the samples measured at 1000 Oe, presented as a function of temperature in Fig. 4 clearly bifurcate below $43 \mathrm{~K}$. At lower magnetic fields the ZFC curves exhibit a peak structure with a maximum dependent on the magnetic field. At higher magnetic field, $H=10000$ Oe, the ZFC magnetization looses its peak structure and increases continuously with decrease in temperature (Fig. 4, inset). The ZFC magnetization versus temperature for the 0.7 at. $\%$ Mn sample detected at 50, 100 and 1000 Oe exhibits the maxima at $42.4,41.4$ and $38.4 \mathrm{~K}$ respectively (Fig. 7a). Fig. 7b presents the ZFC magnetization versus temperature for the 3 at.\% Mn sample recorded at 50, 100 and 1000 Oe showing the maxima at $40.4,40.3$ and $39.7 \mathrm{~K}$ respectively. As can be seen, there is a systematic decrease of the temperature of the maximum in the ZFC magnetization for both the samples in the vicinity of $40 \mathrm{~K}$ with increase in magnetic field. The first derivative $\partial(M T) / \partial T$ for the 3 at.\% Mn sample measured at 1000 Oe (Fig. 6) exhibits a maximum and a minimum at the positions $(38.4 \pm 0.5) \mathrm{K}$ and $(43.0 \pm 0.5) \mathrm{K}$ respectively and these characteristic temperatures were recorded within the limit of accuracy in measurements for all applied magnetic fields. The position of the minimum at $43 \mathrm{~K}$ corresponds to the Curie temperature of the bulk tetragonal $\mathrm{Mn}_{3} \mathrm{O}_{4}, T_{C(\text { bulk })}=$ $42 \mathrm{~K}$ [14], as well as of $\mathrm{Mn}_{3} \mathrm{O}_{4}$ nanoparticles of different sizes where the Curie temperature was found to vary from $40.5 \mathrm{~K}$ up to $44.3 \mathrm{~K}$ [15-17]. The first derivative $\partial(M T) / \partial T$ for the 0.7 at.\% Mn sample measured at 1000 Oe (Fig. 5) shows a maximum and a minimum at the positions $(38.4 \pm 0.5) \mathrm{K}$ and $(42.4 \pm 0.5) \mathrm{K}$ respectively.

The temperature dependence of the real component of the AC magnetic susceptibility for the sample with 3 at.\% Mn measured in the frequency range $1-1488 \mathrm{~Hz}$ is shown in Fig. 8. The real component $\chi^{\prime}$ has a maxi- mum at a temperature $T_{m}$ which has no noticeable shift with increasing frequency for lower frequencies up to 30 $\mathrm{Hz}$ where $T_{m}=40.4 \mathrm{~K}$. For higher frequencies up to $1488 \mathrm{~Hz} T_{m}$ is shifted towards higher temperatures for about $0.3 \mathrm{~K}$. Magnetic field dependent temperature of the maximum in the ZFC magnetization for both the samples and frequency dependent real component of the AC magnetic susceptibility of the 3 at. $\%$ Mn sample, which contains smaller Mn-rich nanoparticles with sizes 3-10 nm, suggest that the temperature $T_{m}$ could be the blocking temperature of the $\mathrm{Mn}_{3} \mathrm{O}_{4}$ nanoparticles which appears just below the Curie temperature $T_{\mathrm{C}}=43 \mathrm{~K}$. Thermally activated blocking process of the magnetic moments of the small $\mathrm{Mn}_{3} \mathrm{O}_{4}$ nanoparticles in our samples is quite possible because superparamagnetic threshold volume at $T=43 \mathrm{~K}$ is $V_{t h}=1.5 \times 10^{-19} \mathrm{~cm}^{3}$, and for spherical particles this volume corresponds to the particle diameter $\mathrm{D}_{t h} \approx 7 \mathrm{~nm}$, as was already reported in Ref. 16. In distinction from our result, in the recently published paper [18] the behavior of an ensemble of the $\mathrm{Mn}_{3} \mathrm{O}_{4}$ nanoparticles is interpreted as a superparamagnetic blocking with the blocking temperature which can be, depending on the particle size, considerably lower than $T_{\mathrm{C}}$.

A comparison of the FM component of magnetization in our sample with 3 at.\% Mn obtained from the $M(H)$ data at $30 \mathrm{~K}$ (not shown), where it was possible to subtract the linear component from the total magnetization, with the literature data $[14,15]$ indicated the content of $\mathrm{Mn}_{3} \mathrm{O}_{4}$ in this sample to be about 0.2 wt.\%. Taking into account the weight percent of manganese in the 3 at.\% Mn sample determined by atomic absorption method as 2.734 wt.\%, we estimated the content of the $\lambda-\mathrm{MnO}_{2}$ phase to be about 4 wt. $\%$. It is obvious that ferrimagnetism of the small amount of $\mathrm{Mn}_{3} \mathrm{O}_{4}$ is strong enough at lower temperatures to mask the relatively weak magnetism of $\lambda-\mathrm{MnO}_{2}$ with AFM interactions. On the other hand, paramagnetic component of the magnetization originating from $\lambda-\mathrm{MnO}_{2}$ overcomes paramagnetic contribution of the $\mathrm{Mn}_{3} \mathrm{O}_{4}$ phase observed in the hightemperature magnetic susceptibility.

\section{Conclusion}

Magnetic properties of the samples with 0.7 and 3 at.\% $\mathrm{Mn}$ incorporated in an amorphous $\mathrm{SiO}_{2}$ matrix have been studied. TEM analysis has shown that the samples contain agglomerates of amorphous silica particles $10-20 \mathrm{~nm}$ in size. In the amorphous matrix two types of nanocrystalline Mn-rich particles are dispersed: smaller Mn-rich nanocrystals with dimensions between 3 and $10 \mathrm{~nm}$, and larger crystalline areas consisting of aggregates of the smaller nanoparticles. At higher temperatures dominant magnetic phase is $\lambda-\mathrm{MnO}_{2}$. At temperatures below $T_{\mathrm{C}}=43 \mathrm{~K}$ strong ferrimagnetism originating from the minor $\mathrm{Mn}_{3} \mathrm{O}_{4}$ phase masks the relatively weak magnetism of $\lambda-\mathrm{MnO}_{2}$ with AFM interactions. Magnetic field dependent ZFC magnetization of both the samples and 
frequency shift of the real component of the AC susceptibility in the 3 at.\% Mn sample suggest that the magnetic moments of the smaller $\mathrm{Mn}_{3} \mathrm{O}_{4}$ nanoparticles are subject to thermally activated blocking process just below the Curie temperature $T_{\mathrm{C}}$. Appearance of the maximum in the ZFC magnetization of the samples below $10 \mathrm{~K}$ indicates spin glass freezing of the magnetic moments at low temperatures which probably occurs in the geometrically frustrated $\mathrm{Mn}$ sublatice of the $\lambda-\mathrm{MnO}_{2}$ crystal structure.

\section{Acknowledgments}

The Ministry of Science and Technological Development of the Republic of Serbia has financially supported this work, Project No 172026.

\section{References}

[1] G.H. Lee, S.H. Huh, J.W. Jeong, B.J. Choi, S.H. Kim, H.C. Ri, J. Am. Chem. Soc. 124, 12094 (2002) and references therein.

[2] M.M. Thackeray, Prog. Solid St. Chem. 25, 1 (1997).

[3] M. Wakihara, Materials Science and Engineering R33, 109 (2001).

[4] P.G. Bruce, B. Scrosati, J.M. Tarascon, Angew. Chem. Int. Ed. 47, 2930 (2008).

[5] J.E. Greedan, N.P. Raju, A.S. Wills, C. Morin, S.M. Shaw, J.N. Reimers, Chem. Mater. 10, 3058 (1998).
[6] Young-II Jang, B. Huang, F.C. Chou, D.R. Sadoway, Y.M. Chiang, J. Appl. Phys. 87, 7382 (2000).

[7] A.S. Wills, N.P. Raju, J.E. Greedan, Chem. Mater. 11, 1510 (1999).

[8] J.R. Martínez, S. Palomares-Sánchez, G. OrtegaZarzosa, F. Ruiz, Y. Chumakov, Materials Letters 60, 3526 (2006).

[9] A. Sundaresan, R. Bhargavi, N. Rangarajan, U. Siddesh, C.N.R. Rao, Phys. Rev. B 74, 161306(R) (2006).

[10] D.W. Abraham, M.M. Frank, S. Guha, Appl. Phys. Lett. 87, 252502 (2005).

[11] F. Luo, W. Song, C.H. Yan, Chem. Phys. Lett. 431, 337 (2006).

[12] A.M.A. Hashem, H.A. Mohamed, A. Bahloul, A.E. Eid, C.M. Julien, Ionics 14, 7 (2008).

[13] N. Wang, Y. Gao, J. Gong, X. Ma, X. Zhang, Y. Guo, L. Qu, Eur. J. Inorg. Chem. 3827 (2008).

[14] K. Dwight, N. Menyuk, Phys. Rev. 119, 1470 (1960).

[15] N. Wang, L. Guo, L. He, X. Cao, C. Chen, R. Wang, S. Yang, Small 3, 606 (2007).

[16] E. Winkler, R.D. Zysler, D. Fiorani, Phys. Rev. B 70, 174406 (2004).

[17] Y.Q. Chang, X.Y. Xu, X.H. Luo, C.P. Chen, D.P. Yu, J. Cryst. Growth 264, 232 (2004).

[18] R.J. Tackett, J.G. Parsons, B.I. Machado, S.M. Gaytan, L.E. Murr, C.E. Botez, Nanotechnology, 21, 365703 (2010). 